\title{
Are food and drink retailers within National Health Service (NHS) venues adhering to NICE guidance on childhood obesity?
}

\author{
A. James ${ }^{1}$, L. Birch ${ }^{2}$, P. Fletcher ${ }^{3}$, S. Pearson ${ }^{4}$, C. Boyçe ${ }^{4}$, A.R. Ness ${ }^{2}$, J. Hamilton-Shield ${ }^{2}$ and \\ F.E. Lithander ${ }^{2}$ \\ ${ }^{1}$ Faculty of Health Sciences, University of Bristol, BS8 1TH, \\ ${ }^{2}$ NIHR Bristol Biomedical Research Centre (Nutrition Theme), BS2 $8 H W$, \\ ${ }^{3}$ Department of General and Old Age Medicine, Cheltenham General Hospital, GL53 7AN and \\ ${ }^{4}$ Department of Clinical Strategy, Cheltenham General Hospital, GL53 7 AN
}

NICE Quality Standard 94, entitled "Obesity in children and young people: prevention and lifestyle weight management programmes"(1), identifies NHS venues as important settings in which to implement childhood obesity prevention strategies. Children may attend an NHS venue as a patient or a visitor where they can be directly or indirectly influenced by the consumer nutrition environment $^{(2-4)}$. The aim of this study was to measure the compliance of two NHS District General Hospitals in England to NICE Quality Standard 94.

Adherence to Quality Statement 1 (QS1) was assessed using the Nutrient Profiling Model (NPM) ${ }^{(5)}$ to classify foods and drinks available in vending machines in the hospitals as either healthy or less healthy. Compliance with Quality Statements 2 and 3 (QS2 \& QS3) was assessed through the measurement of how clearly the shops, cafés and restaurants displayed nutrition information on menus, and the availability and prominent display of healthy foods and drinks in retail outlets, respectively. An assessment tool was also developed to guide the assessment of the consumer nutrition environment.

\begin{tabular}{lccl}
\hline & Mean & SD & Range \\
\hline Crisps, $n 10$ & $10 \cdot 3$ & $4 \cdot 3$ & 1,17 \\
Chocolate, $n$ 19 & $25 \cdot 6$ & $1 \cdot 3$ & 23,27 \\
Sweets, $n$ 2 & $15 \cdot 5$ & $0 \cdot 7$ & 15,16 \\
Sweet \& savoury biscuits, $n 4$ & $19 \cdot 8$ & $6 \cdot 7$ & 10,25 \\
Dried fruit and/or nuts, $n$ 5 & $6 \cdot 4$ & $12 \cdot 8$ & $-8,25$ \\
Drinks, $n$ 15 & $0 \cdot 5$ & $1 \cdot 6$ & $-4,2$ \\
\hline
\end{tabular}

The Table outlines NPM scores for the foods and drinks available in the vending machines. A food is classified as less healthy where it scores $\geq 4$ points and a drink is classified as less healthy where it scores $\geq 1$ point. Adherence to QS1 was poor. Of the 18 vending machines assessed, only $7(39 \%)$ served both a healthy food and a healthy drink. Of the 40 foods available across the vending machines, $36(90 \%)$ were classified as less healthy with a mean NPM score of 20.5 (SD 6.8). Half of the vending machine drinks were classified as less healthy. Neither hospital was compliant with QS2. Nutritional information was not available on menus of food providers in either hospital. There was inconsistent compliance with QS3. Healthy food and drink options were prominently displayed in two restaurants, but all shops and cafés prioritised the display of unhealthy items.

In conclusion, neither hospital was consistently compliant with NICE Quality Standard 94. The lack of availability of healthy foods and drinks, the absence of nutrition information on the menus, the lack of advertising and display of healthy items, and the consistent advertising and prominent display of unhealthy foods and drinks highlights the improvements that are required in NHS venues such as hospitals.

1. NICE (2015) https://www.nice.org.uk/guidance/qs94

2. Black C, Ntani G, Inskip H et al. (2014) Int J Behav Nutr Phys Act 11, 69

3. Caspi CE, Sorensen G, Subramanian SV et al. (2012) Health Place 18, 1172-1187.

4. Holsten JE (2009) Public Health Nutr 12, 397-405.

5. Department of Health (2011) https://www.gov.uk/government/publications/the-nutrient-profiling-model 